\title{
Paclitaxel Impairs Adipose Stem Cell Proliferation and Differentiation
}

Rachel L. Choron MD, ${ }^{\mathrm{a}}$ Shaohua Chang PhD, ${ }^{\mathrm{b}}$ Sophia Khan MD, ${ }^{\mathrm{c}}$ Miguel A. Villalobos MD, ${ }^{\mathrm{d}}$ Ping Zhang $\mathrm{PhD},{ }^{\mathrm{e}}$ Jeffrey P. Carpenter MD, ${ }^{\mathrm{f}}$ Thomas N. Tulenko PhD, ${ }^{\mathrm{g}}$ Yuan Liu MD. ${ }^{\mathrm{h}}$

${ }^{a}$ Department of Surgery, Cooper University Hospital

1 Cooper Plaza Suite 411, Camden, NJ, USA, 08103, Rachel.Choron@gmail.com

${ }^{\mathrm{b}}$ Department of Surgical Research, Cooper University Hospital

401 Haddon Ave, Camden, NJ, USA, 08103, Chang-Shaohua@ cooperhealth.edu

${ }^{\mathrm{c}}$ Department of Surgery, Cooper University Hospital

1 Cooper Plaza Suite 411, Camden, NJ, USA, 08103, Khan-Sophia@ cooperhealth.edu

${ }^{\mathrm{d}}$ Department of Surgery, Cooper University Hospital

1 Cooper Plaza Suite 411, Camden, NJ, USA, 08103, Villalobos-Miguel@ cooperhealth.edu

${ }^{\mathrm{e}}$ Department of Surgical Research, Cooper University Hospital

401 Haddon Ave, Camden, NJ, USA, 08103, Zhang-Ping@ cooperhealth.edu

${ }^{\mathrm{f}}$ Department of Surgery, Cooper University Hospital

1 Cooper Plaza, Camden, NJ, USA, 08103, Carpenter-Jeffrey@ cooperhealth.edu

${ }^{g}$ Department of Surgical Research, Cooper University Hospital

401 Haddon Ave, Camden, NJ, USA, 08103, Tulenko-Thomas@ cooperhealth.edu

${ }^{\text {h }}$ Department of Plastic Surgery, Cooper University Hospital

1 Cooper Plaza Suite 411, Camden, NJ, USA, 08103, YuanLiuMD@gmail.com

Author Contributions: All authors have made substantial contributions to all of the following: (1) the conception and design of the study, or acquisition of data, or analysis and interpretation of data, (2) drafting or revising it critically for important intellectual content, (3) final approval of the version to be submitted.

\section{Corresponding Author:}

Rachel L. Choron MD

720 Chestnut St, Apt B

Philadelphia, PA, USA, 19106

P: 914-584-8552 F: 856-365-7582

E: Rachel.Choron@gmail.com 


\section{ABSTRACT: \\ BACKGROUND:}

Cancer patients with chemotherapy-induced immunosuppression have poor surgical site wound healing. Prior literature supports the use of human adipose-derived stem cell (hASC) lipoinjection to improve wound healing. It has been established multipotent hASCs facilitate neovascularization, accelerated epithelialization, and wound closure in animal models. While hASC wound therapy may benefit surgical cancer patients, the chemotherapeutic effects on hASCs are unknown. We hypothesized Paclitaxel, a chemotherapeutic agent, impairs hASC growth, multipotency, and induces apoptosis.

\section{METHODS:}

hASCs were isolated and harvested from consented, chemotherapy and radiation naïve patients. Growth curves, MTT, and EdU assays measured cytotoxicity and proliferation. Oil-Red-O stain, Alazarin-Red stain, Matrigel tube-formation assay, and qPCR analyzed hASC differentiation. Annexin V assay measured apoptosis. Immunostaining and Western blot determined TNF- $\alpha$ expression.

\section{RESULTS:}

hASCs were selectively more sensitive to Paclitaxel $(0.01 \mu \mathrm{M}-30 \mu \mathrm{M})$ than fibroblasts $(p<0.05)$. After 12 days, Paclitaxel caused hASC growth arrest whereas control hASCs proliferated $(p=0.006)$. Paclitaxel caused an $80.6 \%$ reduction in new DNA synthesis $(p<0.001)$. Paclitaxel severely inhibited endothelial differentiation and capillary-like tube formation. Differentiation markers LPL (adipogenic), alkaline phosphatase (osteogenic), 
CD31 and vWF (endothelial) were significantly decreased (all: $p<0.05$ ) confirming Paclitaxel impaired differentiation. Paclitaxel was also found to induce apoptosis and TNF- $\alpha$ was up-regulated in Paclitaxel-treated hASCs $(p<0.001)$.

\section{CONCLUSION:}

Paclitaxel is more cytotoxic to hASCs than fibroblasts. Paclitaxel inhibits hASC proliferation, differentiation, and induces apoptosis, possibly through the TNF- $\alpha$ pathway. Paclitaxel's severe inhibition of endothelial differentiation indicates neovascularization disruption, possibly causing poor wound healing in cancer patients receiving chemotherapy.

KEYWORDS: Human adipose-derived stem cells, Paclitaxel, wound healing, neovascularization, cancer therapy, chronic wounds 


\section{INTRODUCTION:}

Non-healing surgical wounds are a significant problem. Cancer patients with chemotherapy-induced immunosuppression are at higher risk for poor surgical site wound healing [1-3]. Numerous approaches to improve wound healing have included treatment with transforming growth factor- $\beta$ (TGF- $\beta$ ), fibroblast growth factor (FGF), plateletderived growth factor (PDGF), and epidermal growth factor (EGF) [4-8]. Success with these approaches has been limited secondary to cost, poor efficacy, and the need for continual application of growth factors.

Several studies have revealed advantages of exogenous adult mesenchymal stem cells (MSCs), primarily from bone marrow (BMSCs), to improve wound healing [9-11]. MSCs use paracrine signaling to increase cell migration, proliferation, surrounding cell survival, and accelerate wound healing [9, 12]. However limitations to MSCs include decreased differentiation potential with increased donor age and donor morbidity secondary to bone marrow isolation for MSC access [13, 14].

More recently, the use of human adipose-derived stem cells (hASCs) has emerged as an encouraging alternative to MSCs. An advantage of hASCs is ease of harvest via lipoaspiration causing less donor site morbidity [14,15]. Additional advantages include large quantities of available stem cells given adequate adipose tissue donor sites with less patient discomfort and decreased effect of age [14-17]. 
Like BMSCs, hASCs are multipotent stem cells that play an important role in wound healing and regenerative medicine [18]. Prior literature demonstrated hASC lipoinjection into wounds increases paracrine cytokine secretion facilitating angiogenesis allowing for neovascularization, accelerated epithelialization, and quickened wound closure in animal models [19-21]. In vivo, hASCs have successfully been used as an adjunct to lipoinjection to create an autologous filler to improve soft tissue defects [22-25].

The animal and human studies discussed above show accelerated wound healing and improved soft tissue defects after hASC therapy and they reveal considerable potential for hASC therapy in human patients post-operatively to improve surgical wound healing. Figure 1 illustrates the sequence of procedures used to obtain a hASC line usable for clinical applications. Cancer patients can have significant wound-healing morbidity secondary to chemotherapy-induced immunosuppression [1-3]. The use of hASCs in these immunosuppressed patients could accelerate non-healing surgical wounds. While hASCs may benefit these patients, the chemotherapeutic effects on hASCs are unknown.

Paclitaxel (Figure 2) is a commonly used chemotherapeutic agent in breast and ovarian cancer [26, 27]. Paclitaxel disrupts microtubule organization causing mitotic arrest and induces cancer cell death via apoptosis [28-31]. We focused our current study on Paclitaxel because it is commonly used to treat breast cancer, which occurs in 1 of every 8 women and accounted for 232,340 new cases in 2013 in the United States [32]. Secondly, prior literature has established the successful use of hASCs in breast tissue reconstruction and augmentation [22]. Therefore, there is a large population of 
immunosuppressed breast cancer patients treated with Paclitaxel who could benefit from hASC surgical wound therapy. Our primary study objective was to evaluate the chemotherapeutic effects of Paclitaxel on hASCs in vitro. We hypothesized Paclitaxel impairs hASC cell growth, multipotency, and induces apoptosis.

\section{METHODS:}

\section{Subjects:}

Human adipose tissue was collected for hASC harvesting from patients via lipoaspiration during reconstructive procedures. Informed consent was obtained from healthy patients who had not received prior chemotherapy or radiation. Human lung fibroblasts (WI-38) were obtained from the American Type Culture Collection (ATCC). This experimental protocol was approved by the Institutional Review Board for Human Protection of Cooper University Hospital.

\section{hASC Isolation and Cell Culture (Figure 1) [33]:}

Adipose tissue from liposuction aspirate was rinsed with phosphate buffered saline (PBS) and incubated with $0.1 \%$ collagenase (Worthington) for 1 hour at $37^{\circ} \mathrm{C}$ with vigorous shaking. After centrifugation (1000xg), the top lipid layer was discarded and the remaining liquid was centrifuged again. The residual cell pellet was treated with water to lyse red blood cells, washed with M-199 medium, and recentrifuged. The final cell pellet was plated into T75 cell culture flasks with M-199 containing 10\% fetal bovine serum (FBS) and incubated at $37^{\circ} \mathrm{C}$ in 5\% $\mathrm{CO} 2$ and $95 \%$ room air. After 24 hours, the medium 
was changed to remove non-adherent cells. Passage one or passage two cells were used in this study. WI-38 fibroblasts were grown in DMEM containing 10\% FBS.

\section{MTT Assay:}

A MTT assay was used to assess Paclitaxel cytotoxicity on hASCs and control fibroblast cells. Cultured hASCs and fibroblasts were plated on 24-well plates. Dimethyl sulfoxide (DMSO) created Paclitaxel dilutions: $0.01 \mu \mathrm{M}, 0.1 \mu \mathrm{M}, 1 \mu \mathrm{M}, 3 \mu \mathrm{M}, 10 \mu \mathrm{M}$, and $30 \mu \mathrm{M}$. These dilutions and a DMSO control were added to hASC and fibroblast well plates and incubated for 24 hours or 3 days as indicated. 1x MTT solution $(0.05 \mathrm{mg} / \mathrm{mL})$ was added, incubated, and removed after 2 hours. $200 \mu \mathrm{L}$ of DMSO was added and the SpectraMax M3 (Molecular Devices) obtained optical density readings at 570nm. The relative MTT absorption was calculated and graphed.

\section{Standard Growth/Death Curves:}

Standard growth curves were used to assess hASC proliferation with and without Paclitaxel treatment. hASC lines were plated on 24-well plates in M199 culture medium at $1 \times 10^{4} \mathrm{hASC}$ per well and incubated at $37^{\circ} \mathrm{C}$ with $5 \% \mathrm{CO}$. Culture media which was changed every three days. The average cell count of 3 wells was taken as $n=1$ for each time point.

\section{DNA Incorporation Assay:}

Click-iT® EdU Alexa Fluor® 488 Imaging Kit (Molecular Probes) assessed DNA synthesis during hASC proliferation according to manufacturer's instructions. Green 
fluorescence (Alexa Fluor 488) labeled newly synthesized nuclei, which were examined by a FV1000 laser scanning confocal microscope and photographed with FluoView 1000 software (Olympus, Tokyo, Japan). Three hASC lines were examined in triplicate and 5 images were randomly taken from each sample to calculate the average number of newly synthesized nuclei.

\section{hASC Differentiation:}

Control hASCs and $1 \mu \mathrm{M}$ Paclitaxel-treated hASCs were tested for their ability to differentiate into adipocytes, osteocytes, and endothelial-like cells. hASCs were cultured in M-199 (non-differentiating medium), adipogenic (Invitrogen), osteogenic (Invitrogen), or endothelial differentiation medium (EGM2, Lonza). Adipogenic differentiated cells were fixed for Oil Red O (Sigma) staining to identify lipid droplets in adipose cells. Osteogenic differentiated cells were fixed for Alazarin Red (Sigma) staining to reveal calcium deposition by osteocytes.

\section{In vitro Angiogenesis Assay:}

Matrigel-based tube formation assessed the ability of endothelial-like cells (differentiated from hASCs) to form tubular precapillary-like structures. Control hASCs and $1 \mu \mathrm{M}$ Paclitaxel-treated hASCs were incubated for 3 weeks in endothelial basal medium with supplements. Pre-chilled 24-well plates coated with growth factor-reduced Matrigel (BD Biosciences) were polymerized. $200 \mu \mathrm{L}$ cell suspension was added into Matrigel coated 24-well plates and incubated at $37^{\circ} \mathrm{C}$ with $5 \% \mathrm{CO} 2$. After 24 hours, bright field microscopy was used for observation. Four hASC lines were tested in duplicate. 


\section{RNA Isolation and Real Time-Polymerase Chain Reaction (qRT-PCR):}

Total RNA was isolated from cultured hASCs using Trizol reagent (Life Technologies) according to manufacturer's protocol. Reverse transcription was performed by SuperScriptTM II Reverse Transcriptase Kit (Life Technologies). Real-Time PCR (7500 fast, Applied Biosystem) evaluated the expression level of differentiation marker genes in control hASCs and $1 \mu \mathrm{M}$ Paclitaxel-treated hASCs. Differentiation markers were osteopontin and alkaline phosphatase (osteogenic markers), lipoprotein lipase (LPL) and peroxisome proliferator-activated receptor gamma (PPAR- $\gamma$ ) (adipogenic markers), and van Willebrand factor (vWF), platelet endothelial cell adhesion molecule (CD31), and endothelial nitric oxide synthase (eNOS) (endothelial markers). Glyceraldehyde 3phosphate dehydrogenase (GAPDH), the housekeeping gene, normalized target gene expression. The relative expression of each differentiation marker was calculated and graphed by comparing the fold change in the markers' mRNA expression of differentiated control hASCs and differentiated Paclitaxel-treated hASCs as compared to undifferentiated control hASCs cultured in M-199 medium.

\section{Annexin V Apoptosis Assay:}

The Annexin V apoptosis detection kit (BD Biosciences) using flow cytometry determined if Paclitaxel-treated hASCs undergo apoptosis as compared to control hASCs. Confluent hASCs were treated with $1 \mu \mathrm{M}$ Paclitaxel for 1,3 , or 7 days. Each sample was run on the flow cytometer Accuri C6 (BD Biosciences). These results were graphed to depict the percentage of apoptotic hASCs. 


\section{Western Blot Analysis:}

Protein samples from cultured hASCs were isolated by using an extraction buffer [50 $\mathrm{mM}$ Tris (pH 6.8), 20\% glycerol, 1\% SDS, and a protease inhibitor cocktail (Sigma)]. Protein concentration was determined by DC protein assay kit (Bio-Rad). 40 $\mu \mathrm{g}$ total protein from each sample was loaded to $4-20 \%$ precast gels running at $120 \mathrm{~V}$ and transferred to polyvinylidene difluoride (PVDF) membranes at $4{ }^{0} \mathrm{C}$. After 24 hours, the membranes were blocked with $5 \%$ bovine serum albumin blocking buffer and incubated with the primary antibody: either TNF- $\alpha$ (ABCam) at 1:500 dilution or $\beta$-actin (Sigma) at 1:5,000 dilution. After 24 hours the corresponding secondary antibodies were applied at 1:3,000 dilutions for TNF- $\alpha$ and 1:10,000 dilutions for $\beta$-actin. After 1 hour in the dark followed by washing, target protein bands were visualized and quantified by scanning with Fluo Chemi Q imager (Protein Simple).

\section{Immunofluorescence:}

Immunofluorescent staining was used to depict TNF- $\alpha$ in $1 \mu \mathrm{M}$ Paclitaxel-treated hASCs as compared to control hASCs. Confluent hASCs were fixed using $4 \%$ formaldehyde, washed with PBS, permeabilized, and blocked in a buffer containing $0.2 \%$ triton X-100 and $5 \%$ FBS. The primary antibody (TNF- $\alpha$ ) was added followed by the secondary antibody. After washings, hASCs were examined by FV1000 laser scanning confocal microscope and photographed under a FluoView 1000 software (Olympus, Tokyo, Japan). 


\section{Data Analysis:}

All experiments were performed with three to seven hASC lines from different patients. The quantitative data was presented as mean \pm standard error. Student's t-test and the Wilcoxon rank-sum test were used to assess statistical significance. A $p$ value of $\leq 0.05$ was considered significant for all experiments.

\section{RESULTS:}

\section{Cytotoxicity of Paclitaxel on hASCs and Fibroblasts:}

Five hASC lines and 4 fibroblast cell lines used in MTT assays assessed cell viability after Paclitaxel treatment (Figure 3A). MTT assays did not reveal a Paclitaxel dosedependent response in hASCs or fibroblasts (Paclitaxel concentrations: $0.01 \mu \mathrm{M}, 0.1 \mu \mathrm{M}$, $1 \mu \mathrm{M}, 3 \mu \mathrm{M}, 10 \mu \mathrm{M}$, and $30 \mu \mathrm{M})$. However even the lowest dose $(0.01 \mu \mathrm{M})$ proved cytotoxic to hASCs. hASCs were selectively more cytotoxic to Paclitaxel than fibroblasts, as shown in Figure 3. After 24 hours of Paclitaxel, both $0.01 \mu \mathrm{M}$ and $1 \mu \mathrm{M}$ doses of Paclitaxel were significantly more cytotoxic to hASCs than fibroblasts $(p=0.02$, $p=0.008$; Figure 3A). Additionally MTT assays using 7 hASC lines and 6 fibroblast cell lines assessed cell viability after 3 days of Paclitaxel treatment (Figure 3B). hASCs were again selectively more cytotoxic to Paclitaxel than fibroblasts over the longer time period $(0.1 \mu \mathrm{M} p=0.025,1 \mu \mathrm{M} p=0.011,3 \mu \mathrm{M} p=0.01$, and $30 \mu \mathrm{M} p=0.045$; Figure $3 \mathrm{~B})$.

\section{Paclitaxel's Effect on hASC Proliferation:}

Four hASC lines were used to study $1 \mu \mathrm{M}$ Paclitaxel on hASC proliferation over a 12-day culture period. The resulting growth curves (Figure 4A) demonstrated control hASCs 
grew from $1.0 \times 10^{4}$ hASCs to $9.96 \times 10^{4} \pm 2.46 \times 10^{4}$ hASCs over 12 days. Where as Paclitaxel-treated hASCs demonstrated complete growth arrest. $1 \mu \mathrm{M}$ Paclitaxel treatment abrogated hASC proliferation within 24 hours. $1.0 \times 10^{4}$ hASCs were plated and only $0.99 \times 10^{4} \pm 0.10 \times 10^{4}$ hASCs remained after 12 days $(p=0.006$; Figure $4 \mathrm{~A})$.

EdU assays assessed proliferation with three hASC lines. Figure 4B depicts Paclitaxeltreated hASCs had less stained cell nuclei indicating less DNA synthesis than control hASCs. Paclitaxel-treated hASCs had an 80.6\% decrease in DNA synthesis (Figure 4B and 4 C). After 3 days, control hASCs had $65.3 \% \pm 4.0 \%$ EdU DNA incorporation whereas Paclitaxel-treated hASCs only had $12.7 \% \pm 3.1 \%$ DNA incorporation $(p<0.001$; Figure 4C).

\section{Paclitaxel's Effect on hASC Adipogenic and Osteogenic Differentiation:}

Paclitaxel inhibited hASC differentiation into adipocytes and osteocytes. Figure 5 demonstrates Paclitaxel's effect on 5 hASC lines' differentiation after 3 weeks in adipogenic or osteogenic induction medium. Oil Red O stain demonstrated less stained lipid droplets in Paclitaxel-treated adipogenic-differentiated hASCs compared to control hASCs (Figure 5A). Alazarin red staining reflected calcium deposition in osteogenicinduced hASCs treated with and without Paclitaxel (Figure 5B). Paclitaxel did not affect a change in calcium deposition.

Real-time PCR confirmed Paclitaxel inhibited hASC differentiation. The relative expression of each differentiation marker was graphed by comparing the fold change in 
the markers' mRNA expression of differentiated control hASCs vs. Paclitaxel-treated hASCs as compared to undifferentiated control hASCs cultured in M-199 medium. The expression of adipogenic markers was examined with $5 \mathrm{hASC}$ lines. LPL expression was significantly reduced in Paclitaxel-treated hASCs compared to control hASCs $(779 \pm 485$ vs. $207 \pm 128, p=0.043)$. PPAR- $\gamma$ expression was also reduced $(28.3 \pm 16.8$ vs. $25.4 \pm 20.3$, $p=0.345$; Figure 5C). The expression of osteogenic markers, alkaline phosphatase and osteopontin, were examined in 5 hASC lines. Alkaline phosphatase was significantly reduced in Paclitaxel-treated hASCs compared to control hASCs (11.3 \pm 1.4 vs. 3.3 \pm 1.6 , $p=0.043)$. Osteopontin was also reduced (6.1 \pm 2.6 vs. $3.5 \pm 1.7, p=0.345$; Figure 5D).

\section{Paclitaxel's Effect on hASC Endothelial Differentiation:}

In vitro angiogenesis assays by Matrigel-based tube formation showed Paclitaxel treatment impaired hASC differentiation into pre-capillary endothelial-like cells with tubular polygonal networks (Figure 6A). Control hASCs demonstrated abundant tube formation function after 3 weeks of differentiation in endothelial induction medium. Conversely, Paclitaxel-treated hASCs did not show any tube-like formations. Bright field microscopy revealed a remarkable morphology change in Paclitaxel-treated hASCs (Figure 6A). Four individual hASC lines were tested in duplicate and this phenomenon was highly repeatable.

Real-time PCR showed the expression of endothelial markers, CD31 and vWF, were significantly reduced in Paclitaxel-treated hASCs compared to control hASCs $(18.3 \pm 6.3$ vs. $6.5 \pm 3.1, p=0.016 ; 12.0 \pm 5.4$ vs. $6.1 \pm 3.5, p=0.031$; Figure $6 \mathrm{~B})$. The expression of the 
endothelial marker, eNOS, was reduced in Paclitaxel-treated hASCs compared to control hASCs (222.4 \pm 129.9 vs. $62.8 \pm 43.3, p=0.500$; Figure 6B).

\section{Paclitaxel Induced Apoptosis and Up-Regulation of TNF- $\alpha$ :}

Annexin V apoptosis detection kit using flow cytometry demonstrated more Paclitaxeltreated hASCs enter early apoptosis compared to control hASCs. The majority (78.8\%) of control hASCs were normal, whereas $15.8 \%$ were in early apoptosis (Figure 7A). 24 hour Paclitaxel treatment resulted in $76.0 \%$ living hASCs and $18.8 \%$ early apoptotic hASCs (Figure 7B). As Paclitaxel treatment length increased, the percentage of apoptotic hASCs increased. After continual Paclitaxel treatment over 3 and 7 days, $30.8 \%$ and $64.7 \%$ of hASCs were apoptotic (Figure 7C, 7E). When only one dose of Paclitaxel on day 1 and medium changes without Paclitaxel for 7 days, there were only $51.8 \%$ apoptotic hASCs; with continual Paclitaxel over 7 days, $64.7 \%$ of hASCs were apoptotic (Figure 7D, 7E).

Immunostaining revealed Paclitaxel-treated hASCs had more intensive peri-nuclear TNF$\alpha$ staining compared to control hASCs (Figure 8A). Western blots further identified 3.5fold greater TNF- $\alpha$ protein expression in Paclitaxel-treated hASCs compared to control hASCs $(p<0.001)$. This indicates the up-regulation of TNF- $\alpha$ as the likely mechanism of apoptosis (Figure 8B, 8C).

\section{DISCUSSION:}


This study demonstrates the chemotherapeutic agent Paclitaxel has damaging effects on hASCs. Paclitaxel has selective cytotoxicity to hASCs and is markedly more cytotoxic to hASCs than human lung fibroblasts. Paclitaxel inhibits proliferation and differentiation and induces increasing apoptosis in hASCs over time. Since hASCs are self-renewing and capable of differentiating into other closely related cell phenotypes, this strongly suggests Paclitaxel impairs tissue restoration by hASC function.

\section{Paclitaxel Dosing In Vivo vs. In Vitro:}

Although hASCs were studied in cell culture, Paclitaxel doses were not dissimilar to clinical standard cancer therapy. Paclitaxel dosing in breast cancer is $135-250 \mathrm{mg} / \mathrm{m}^{2}$ over 3-hour infusions every three weeks $[34,35]$. The pharmacokinetics of Paclitaxel in vivo show that patients who received $175 \mathrm{mg} / \mathrm{m}^{2}$ for 3 hour infusion periods had a maximum Paclitaxel plasma concentration of $3.6 \mu \mathrm{g} / \mathrm{mL}$, corresponding to $4.2 \mu \mathrm{M}$ concentration (molecular weight $853.9 \mathrm{~g} / \mathrm{mol}$ ) [36]. Therefore, we tested several doses of Paclitaxel ranging from $0.01 \mu \mathrm{M}-30 \mu \mathrm{M}$. While all the doses proved cytotoxic to hASCs, $1 \mu \mathrm{M}$ Paclitaxel was significantly more cytotoxic to hASCs than fibroblasts after treatment for both 24 hours and 3 days. This correlated with the in vivo systemic levels of Paclitaxel in patients; therefore we used the $1 \mu \mathrm{M}$ Paclitaxel concentration in vitro in all experiments to best correlate with clinical systemic levels.

\section{Influence of Harvest Site on hASC Function:}

Prior literature has revealed hASC lipoaspiration harvest site does not affect cell function [37-39]. In 2011, Jurgens et al. found there was not a difference in chondrogenic or 
osteogenic differentiation ability when comparing abdominal vs, thigh/hip harvest sites for hASCs [37]. In 2013, this was confirmed by Engles et al, who found there was not a functional difference in hASCs isolated from the inguinal, flank, omental, or neck regions, however they did find hASCs from those sites grew better than hASCs derived from the pericardium [39]. While hASC function is not dependent on harvest site, hASC yield has been shown to be higher when derived from the abdominal anatomic site [37, 40]. Unpublished data from our lab also confirmed a higher yield of hASCs from the abdominal site as compared to the thigh or flank. Because the abdominal site provides the best hASC yield, we chose that patient harvest site for our experiment to develop hASC lines. While prior literature indicates future stem cell application for therapeutic uses could use hASCs originating from multiple anatomic locations, we find the abdominal site appealing because of ease of harvest and its high yield.

\section{Wound Healing Mechanisms Effected by Paclitaxel-Treated hASCs:}

Paclitaxel's effects on hASCs may contribute to poor wound healing in chemotherapyreceiving cancer patients. hASC lipoinjection into wounds increases paracrine cytokine secretion facilitating angiogenesis allowing for neovascularization, accelerated epithelialization, and quickened wound closure in animal models [19-21]. Our study revealed the endothelial markers (vWF, CD31, and eNOS) showed endothelial differentiation was significantly inhibited in Paclitaxel-treated hASCs. Additionally the Matrigel angiogenesis assay revealed much less functional capacity of the Paclitaxeltreated differentiated endothelial hASCs to form pre-capillary tube-like structures. Therefore, patients who receive Paclitaxel likely have wound healing inhibition 
secondary to hASCs' inability to neovascularize. In patients who have multiple risk factors for poor wound healing, it maybe advantageous in the future to bank their hASCs preoperatively prior to chemotherapeutic drug exposure for possible therapy in chronic wounds. Therefore postoperatively these patients could potentially undergo hASC wound injection to promote wound healing without the cytotoxic chemotherapy effect.

\section{$\underline{\text { hASC Recovery after Treatment with Paclitaxel: }}$}

The extent to which hASCs can recover after Paclitaxel treatment is unknown and will be examined in future studies. The Annexin V apoptosis detection kit determined more Paclitaxel-treated hASCs entered early apoptosis when continually exposed to Paclitaxel compared to hASCs given a one-time dose $(51.8 \%$ vs. $64.7 \%$; Figure $7 \mathrm{D}, 7 \mathrm{E})$. This suggests hASCs maybe able to partially recover after a finite period of Paclitaxel treatment.

One prior study examined Cisplatin, Vincristine, and Camptothecin's effects on hASCs. They found hASCs were resistant to the chemotherapeutic agents, maintained their stem cell characteristics, and showed recovery after chemotherapeutic drug exposure [41]. These findings were not consistent with Paclitaxel's effects on hASCs. Different chemotherapeutic agents may have different effects on hASCs, secondary to mechanism of action. Therefore, if a cancer patient has a known risk for wound healing morbidity, chemotherapy maybe tailored to avoid Paclitaxel's negative effect.

\section{Future Work: Mechanism of Paclitaxel-Induced Apoptosis of hASCs:}


There are several core apoptotic pathways. One of the most studied death ligands that induce apoptosis via death receptors is TNF- $\alpha$ [42]. Our future work will be centered on the mechanism causing Paclitaxel-induced apoptosis in hASCs. Our preliminary findings have revealed more intensive peri-nuclear TNF- $\alpha$ staining and greater TNF- $\alpha$ protein expression in Paclitaxel-treated hASCs compared to control hASCs. This indicates the up-regulation of TNF- $\alpha$ is a potential mechanism of Paclitaxel-induced apoptosis. Recent literature has supported this theory; Minero et al. revealed the addition of TNF- $\alpha$ to Paclitaxel dosing in hepatocellular carcinoma cells in-vitro and in an in-vivo mouse model induced greater apoptosis than either Paclitaxel or TNF- $\alpha$ alone [43].

While the mechanism of Paclitaxel in other cell lines has been described, our conclusion that Paclitaxel induces apoptosis (possibly secondary to TNF- $\alpha$ ) in hASCs is novel. To our knowledge, there is no literature describing the mechanism of apoptotic action Paclitaxel takes on hASCs or the role TNF- $\alpha$ plays in hASCs. Based on our experiments and prior literature, we hypothesize Paclitaxel promotes TNF- $\alpha$ cytokine activity within the hASC resulting in inhibition of RNA and protein synthesis resulting in apoptosis (Figure 9) [44].

Chemotherapeutic agents are not the only modalities used in cancer patients that impair hASC activity. Radiation therapy is also an integral part of breast cancer treatment [4548], however its use is complicated by side effects, including poor wound healing. While the use of hASCs in cancer patients with wound healing impairment secondary to radiation maybe beneficial, the effects of radiation on hASCs have yet to be studied. 
Currently we are working on a future project comparing hASCs from radiotherapy exposed and unexposed sites in patients undergoing breast reconstruction. Preliminary results have revealed decreased proliferation capacity, differentiation, and gene expression.

\section{Limitations:}

The in vitro design model may not accurately simulate in vivo pathways. While we researched maximum plasma concentration of Paclitaxel in vivo, the specific translation to in vivo plasma concentrations over time is limited. Four to seven hASC lines were used in each experiment; increasing the number of cell lines could reveal variations in our data. Additionally, our in vitro studies examined Paclitaxel's effects over short periods of time (24 hours-3 weeks), whereas Paclitaxel exposure in vivo is longer; long-term effects may not have been observed in this study. Lastly, we did not take into account the co-morbidities of hASC donors and the possible effects those co-morbidities may have on hASC function.

\section{CONCLUSIONS:}

The cytotoxic effect of Paclitaxel on hASCs is selectively higher in comparison to fibroblasts. Paclitaxel inhibits proliferation and differentiation of hASCs into endothelial cells, adipocytes, and osteocytes. Paclitaxel's severe inhibition of endothelial differentiation indicates neovascularization disruption, possibly causing poor wound healing in cancer patients receiving chemotherapy. 


\section{ACKNOWLEDGEMENTS:}

We would like to thank Dr. Spencer Brown, who provided critical editing, advisement, and overall research support to this project. 


\section{REFERENCES:}

1. Springfield DS. Surgical wound healing. Cancer Treat Res. 1993;67:81-98.

2. Drank DB, Oishi SN. Wound healing considerations in chemotherapy and radiation therapy. Clin Plast Surg. 1995;22(1):31-7.

3. Cornell K, Waters DJ. Impaired wound healing in the cancer patient: effects of cytotoxic therapy and pharmacologic modulation by growth factors. Vet Clin North Am Small Anim Pract. 1995;25(1):111-31.

4. Ashraf A, Lee PH, Kim K, Zaporojan V, Bonassar L, Valentini R, et al. Effect of sustained-release PDGF and TGF-beta on cyclophosphamide induced impaired wound healing. Plast Reconstr Surg. 2009;124:1118-24.

5. Obara K, Ishihara M, Fujita M, Kanatani Y, Hattori H, Matsui T, et al. Acceleration of wound healing in healing-imparied $\mathrm{db} / \mathrm{db}$ mice wit ha photocrosslinkable chitosan hydrogel containing fibroblast growth factor-2. Wound Repair Regen. 2005; 13: 390-97. 6. Puolakkainen PA, Twardzik DR, Ranchalis JE, Pankey SC, Reed MJ, Gombotz WR. The enhancement in wound healing by transforming growth factor-beta 1 (TGF-beta 1) depends on the topical delivery system. J. Surg Res. 1995;58:321-9.

7. Steed DL. Modifying the wound healing response with exogenous growth factors. Clin Plast Surg. 1998;25:397-405.

8. Tsang MW, Wong WK, Hung CS, Lai KM, Tang W, Cheung EY, et al. Human epidermal growth factor enhances healing of diabetic foot ulcers. Diabetes Care. 2003;26:1856-61. 
9. Maxon S, Lopez EA, Yoo D, Danilkovitch-Miagkova A, Leroux MA. Concise review: Role of mesenchymal stem cells in wound repair. Stem Cells Transl Med. 2012;1(2):1429.

10. Kode JA, Mukherjee S, Joglekar MV, Hardikar AA. Mesenchymal stem cells: immunobiology and role in immunomodulation and tissue regeneration. Cytotherapy; 2009;11(4):377-91.

11. Manuguerra-Gagne R, Boulos PR, Ammar A, Leblond FA, Krosl G, Pichette V, et al. Transplantation of mesenchymal stem cells promotes tissue regeneration in a glaucoma model through laser-induced paracrine factor secretion and progenitor cell recruitment. Stem Cells. 2013;31(6):1136-48.

12. Fathke C, Wilson L, Hutter J, Kapoor V, Smith A, Hocking A, et al. Contribution of bone marrow-derived cells to skin: Collagen deposition and wound repair. Stem Cells. 2004;22:812-22.

13. Stenderup K, Justesen J, Clausen C, Kassem M. Aging is associated with decreased maximal life span and accelerated senescence of bone marrow stromal cells. Bone. 2003;33:919-26.

14. De Ugarte DA, Morizono K, Elbarbary A, Alfonso Z, Zuk PA, Zuh M, et al. Comparison of multi-lineage cells from human adipose tissue and bone marrow. Cells Tissue Organ. 2003;174:101-9.

15. Kern S, Eichler H, Stoeve J, Kluter H, Bieback K. Comparative analysis of mesenchymal stem cells from bone marrow, umbilical cord blood, or adipose tissue. Stem Cells. 2006;24:1294-301. 
16. Mizuno H, Tobita M, Cagri Uysal A. Concise review: Adipose-derived stem cells as a novel tool for future regenerative medicine. Stem Cells. 2012;30:804-10.

17. Zhang P, Moudgill N, Hager E, Tarola N, Dimatteo C, Mcllhenny S, et al.

Endothelial differentiation of adipose-derived stem cells from elderly patients with cardiovascular disease. Stem Cells Dev. 2011;20(6):977-88.

18. Zuk PA, Zhu M, Ashjian P, De Ugarte DA, Huang JI, Mizuno H, et al. Human adipose tissue is a source of multipotent stem cells. Mol Biol Cell. 2002;13:4279-95. 19. Gimble JM, Bunnell BA, Guilak F. Human adipose-derived cells: An update on the transition to clinical translation. Regen Med. 2012;7(2):225-35.

20. Nie, C. Yang D, Xu J, Si Z, Jin X, Zhang J. Locally administered adipose derived stem cells accelerate wound healing through differentiation and vasculogenesis. Cell Transplant. 2011;20(2):205-16.

21. Zhu M, Zhou Z, Chen Y, Schreiber R, Ransom JT, Fraser JK, et al. Supplementation of fat grafts with adipose-derived regenerative cells improves long-term graft retention. Ann Plast Surg. 2010;64(2):222-8.

22. Yoshimura K, Sato K, Aoi N, Kurita M, Hirohi T, Haril K. Cell assisted lipostransfer for cosmetic breast augmentation: supportive use of adipose-derived stem/stromal cells. Aesthetic Plast Surg. 2008;32(1):48-55.

23. Yoshimura K, Suga H, Eto H. Adipose-derived stem/progenitor cells: roles in adipose tissue remodeling and potential use for soft tissue augmentation. Regen Med. 2009;4:26573. 
24. Tanikawa DY, Aguena M, Bueno DF, Passos-Bueno MR, Alonso N. Fat grafts supplemented with adipose-derived stromal cells in the rehabilitation of patients with craniofacial microsomia. Plast Reconstr Surg. 2013;132(1):141-52.

25. Orbay H, Takami Y, Hyakusoku H, Mizuno H. Acellular dermal matrix seeded with adipose-derived stem cells as a subcutaneous implant. Aesthetic Plast Surg. 2011;35(5):756-63.

26. Khayat D, Antoine EC, Coeffie D. Taxol in the management of cancers of the breast and the ovary. Cancer Invest. 2000;18:242-60.

27. Holmes FA, Walters RS, Theriault RL, Forman AD, Newton LK, Raber MN, et al. Phase II trial of taxol, an active drug in the treatment of metastatic breast cancer. J Natl Cancer Inst. 1991;83:1797-805.

28. Rowinsky EK, Donehower RC. Paclitaxel (taxol). N Engl J Med. 1995;332:1004-14. 29. Torres K, Horwitz SB. Mechanisms of Taxol-induced cell death are concentration dependent. Cancer Research. 1998;58:3620-6.

30. Lu Z, Jia J, Di L, Song G, Yuan Y, Ma B, et al. DNA methyltransferase inhibitor CDA-2 synergizes with high dose Thiotepa and Paclitaxel in killing breast cancer stem cells. Front Biosci (Elite Ed). 2001;3:240-9.

31. Pan Z, Gollahon L. Paclitaxel attenuates Bcl-2 resistance to apoptosis in breast cancer cells through an endoplasmic reticulum-mediated calcium release in a dosage dependent manner. Biochemical and Biophysical Research Communications. 2013;432:431-7.

32. DeSantis C, Ma J, Bryan L, Jemal A. Breast cancer statistics, 2013. CA Cancer J Clin. 2014;64(1):52-62. 
33. Yang XF, He X, He J, Zhang LH, Su XJ, Dong ZY, et al. High efficient isolation and systematic identification of human adipose derived mesenchymal stem cells. J Biomed Sci. 2011;18:59.

34. Perez EA. Paclitaxel in Breast Cancer. The Oncologist. 1998;3:373-89.

35. Seidman AD, Tiersten A, Hudis C, Gollub M, Barrett S, Yao TJ, et al. Phase II trial of Paclitaxel by 3-hour infusion as initial and salvage chemotherapy for metastatic breast cancer. J Clin Oncol. 1995;13:2575-81.

36. Taxol ${ }^{\circledR}$ (Paclitaxel) Injection. Bristol-Myers Squibb Company. Princeton, NJ 08543

USA. 2011; April. Available at: http://packageinserts.bms.com/pi/pi_taxol.pdf

37. Jurgens WJFM, Oedayrajsingh-Varma MJ, Helder MN, ZandiehDoulabi B, Schouten TE, Kuik DJ, et al. Effect of tissue-harvesting site on yield of stem cells derived from adipose tissue: implications for cell-based therapies. Cell Tissue Res. 2008;332(3):41526.

38. Oedayrajsingh-Varma MJ, Van Ham SM, Knippenberg M, Helder MN, Klein-Nulend J, Schouten TE, et al. Adipose tissue-derived mesenchymal stem cell yield and growth characteristics are affected by the tissue-harvesting procedure. Cytotherapy. 2006;8(2):166-77.

39. Engels PE, Tremp M, Kingham PJ, di Summa PG, Largo RD, Schaefer DJ, Kalbermatten DF. Harvest Site Influences the growth properties of adipose derived stem cells. Cytotechnology. 2013;65(3):437-45.

40. Padoin AV, Braga-Silva J, Martins P, Rezende K, Rezende AR, Grechi B, et al. Sources of processed lipoaspirate cells: influence of donor site on cell concentration. Plast Reconstruc Surg. 2008;122(2):614-8. 
41. Liang W, Xia H, Li J, Zhao RC. Human adipose tissue derived mesenchymal stem cells are resistant to several chemotherapeutic agents. Cytotechnology. 2011;63(5):52330.

42. Almasan A, Ashkenazi A. Apo2L/TRAIL: apoptosis signaling, biology, and potential for cancer therapy. Cytokine Growth Factor Rev. 2003;14:337-48.

43. Minero VG, De Stefanis D, Costelli P, Baccino FM, Bonelli G. In vitro and in vivo conditional sensitization of hepatocellular carcinoma cells to TNF-induced apoptosis by Taxol. Cell Cycle. 2015; 7.

44. Baud V, Karin M. Signal transduction by tumor necrosis factor and its relatives. Trends Cell Biol. 2001;11(9):372-7.

45. Clarke M, Collins R, Darby S, et al. Effects of radiotherapy and of differences in the extent of surgery for early breast cancer on local recurrence and 15-year survival: An overview of the randomized trials. Lancet. 2005;366:2087-106.

46. Smith BD, Arthur DW, Buchholz TA, et al. Accelerated partial breast irradiation consensus statement from the American Society for Radiation Oncology (ASTRO). Int $J$ Radiat Oncol Biol Phys. 2009; 74: 987-1001.

47. Nielsen HM, Overgaard M, Grau C, et al. Study of failure pattern among high-risk breast cancer patients with or without postmastectomy radiotherapy in additiona to adjuvant systemic therapy: Long-term results from the Danish Breast Cancer Cooperative Group DBCG 82b and c randomized studies. J Clin Oncol. 2006;24:2268-75.

48. Ragaz J, Olivotto IA, Spinelli JJ, et al. Locoregional radiation therapy in patients with high-risk breast cancer receiving adjuvant chemotherapy: 20-year results of the British 
Columbia randomized trial. J Natl Cancer Inst. 2005;97:116-26. 


\section{FIGURE LEGENDS:}

Figure 1. Establishing human adipose derived stem cell lines (hASC). Starting with patient lipoaspiration, followed by digestion with collagenase and cetrifugation to create the stem cell pellet. After hASC incubation, culture in different differentiation mediums results in adipocyte, osteocyte, and endothelial cell development and the possibility for clinical application such as improving wound healing with hASCs.

Figure 2. The molecular form of Paclitaxel. Paclitaxel is a chemotherapeutic agent often used in breast, ovarian, and lung cancer. It disrupts microtubule organization causing mitotic arrest and induces cancer cell death via apoptosis.

Figure 3. The effect of Paclitaxel on hASC viability. A. Cytotoxicity of Paclitaxel in hASCs and fibroblasts after 1 day of treatment. MTT Assay revealed hASCs were selectively more cytotoxic to Paclitaxel than fibroblasts. Both $0.01 \mu \mathrm{M}$ and $1 \mu \mathrm{M}$ doses of Paclitaxel were significantly more cytotoxic to hASCs than fibroblasts ( $p=0.02$ and $p=0.008)$. B. Cytotoxicity of Paclitaxel in hASCs and fibroblasts after 3 days of treatment. MTT Assay revealed a similar trend to the 1-day treatment; hASCs were selectively more cytotoxic to Paclitaxel than fibroblasts at the following doses: $(0.1 \mu \mathrm{M}$ : $p=0.025,1 \mu \mathrm{M}: p=0.011,3 \mu \mathrm{M}: p=0.01$, and $30 \mu \mathrm{M}: p=0.045)$.

Figure 4. The effect of Paclitaxel (PTX) on hASC growth. A. A cell growth curve of hASCs with and without Paclitaxel treatment. The number of control hASCs increased steadily while Paclitaxel treated hASCs experienced growth arrest after 12 days 
$(p=0.006)$. B. Images after EdU Assay measured DNA incorporation in hASCs with and without Paclitaxel treatment. C. Analyzed DNA incorporation in hASCs with and without Paclitaxel shown by bar graph. Paclitaxel treated hASCs had significantly less proliferation and DNA synthesis than control hASCs as measured by EdU DNA incorporation $(\mathrm{p}<0.001)$.

Figure 5. The effect of Paclitaxel (PTX) on hASCs adipogenic and osteogenic differentiation. A. Adipogenic differentiation. Oil Red O stain showed there were less stained lipid droplets in the adipogenic-induced hASCs treated with Paclitaxel as compared to control hASCs. B. Osteogenic differentiation. Alazarin red stain illustrated the calcium deposits seen in osteogenic-induced hASCs treated with and without Paclitaxel. C. Adipogenic markers, LPL and PPAR- $\gamma$, revealed reduced expression in Paclitaxel treated hASCs. $(p=0.043, p=0.345)$. D. Osteogenic markers, alkaline phosphatase and osteopontin, revealed reduced expression in Paclitaxel treated hASCs. $(p=0.043, p=0.345)$.

Figure 6. The effect of Paclitaxel (PTX) on hASC endothelial differentiation. A. Bright field cell images of control hASCs and Paclitaxel treated hASCs in 6-well plates and Matrigel coated 24-well plates. Light microscopy showed Paclitaxel treatment induced cell morphology changes and less tube formation. B. The expression of endothelial differentiation markers CD31, vWF, and eNOS, measured by Real-time PCR. Two of the three endothelial markers, CD31 and vWF, revealed significantly reduced expression in Paclitaxel treated hASCs $(p=0.016, p=0.031, p=0.500)$. 
Figure 7. The Annexin V apoptosis detection kit using flow cytometry determined an increasing number of Paclitaxel (PTX) treated hASCs entered early apoptosis as compared to untreated control hASCs. A. $15.8 \%$ of control cells in the early apoptotic phase. B. After 24 hours of Paclitaxel treatment, $18.8 \%$ of hASCs were in early apoptosis. C. After 3 days of Paclitaxel treatment, 30.8\% of hASCs were in early apoptosis. D. When 1 dose of Paclitaxel was given, followed by medium changes without Paclitaxel for 7 days, $51.8 \%$ of hASCs were in early apoptosis. E. After 7 days of continual treatment with Paclitaxel, 64.7\% of hASCs were in early apoptosis.

Figure 8. The localization and expression of TNF- $\alpha$ in control and Paclitaxel (PTX) treated hASCs. A. Immunostaining revealed Paclitaxel treated hASCs had an increased amount of TNF- $\alpha$ as compared to control hASCs. B. A representative western blot image for TNF and $\beta$-actin in control and Paclitaxel treated hASCs. C. Analyzed Western blot data for 4 hASC lines, the expression of TNF- $\alpha$ protein was normalized by $\beta$-actin $(p<0.001)$

Figure 9. Human adipose derived stem cell (hASC) function with and without Paclitaxel. A. The natural course of hASC differentiation. B. The inhibiting effect of Paclitaxel on hASC differentiation and Paclitaxel's induction of apoptosis, possibly through TNF- $\alpha$ 's upregulation. 
Figure 1

Figure 1. Human Adipose Derived Stem Cell Line Development and Use

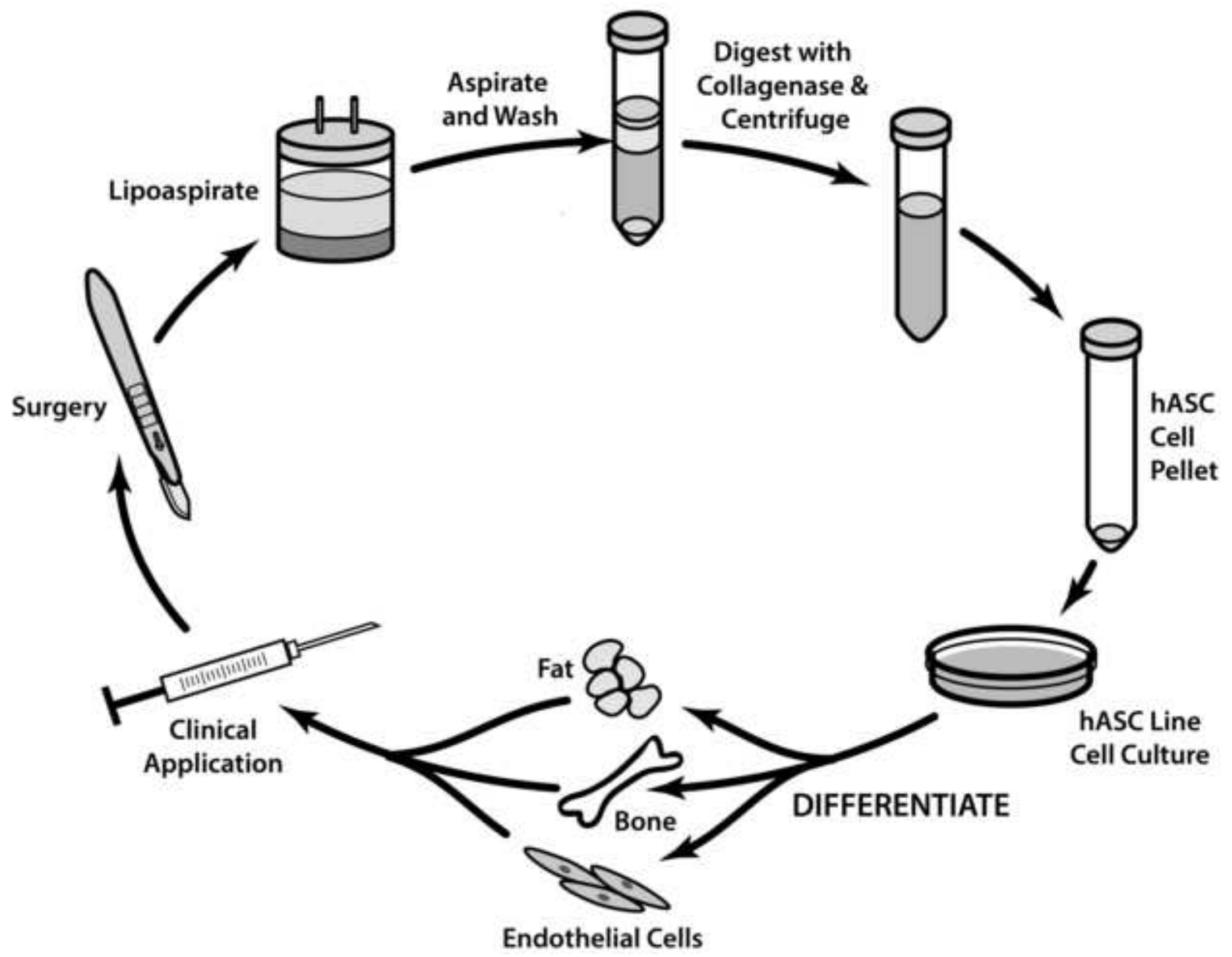


Figure 2. Paclitaxel, a Chemotherapeutic Agent

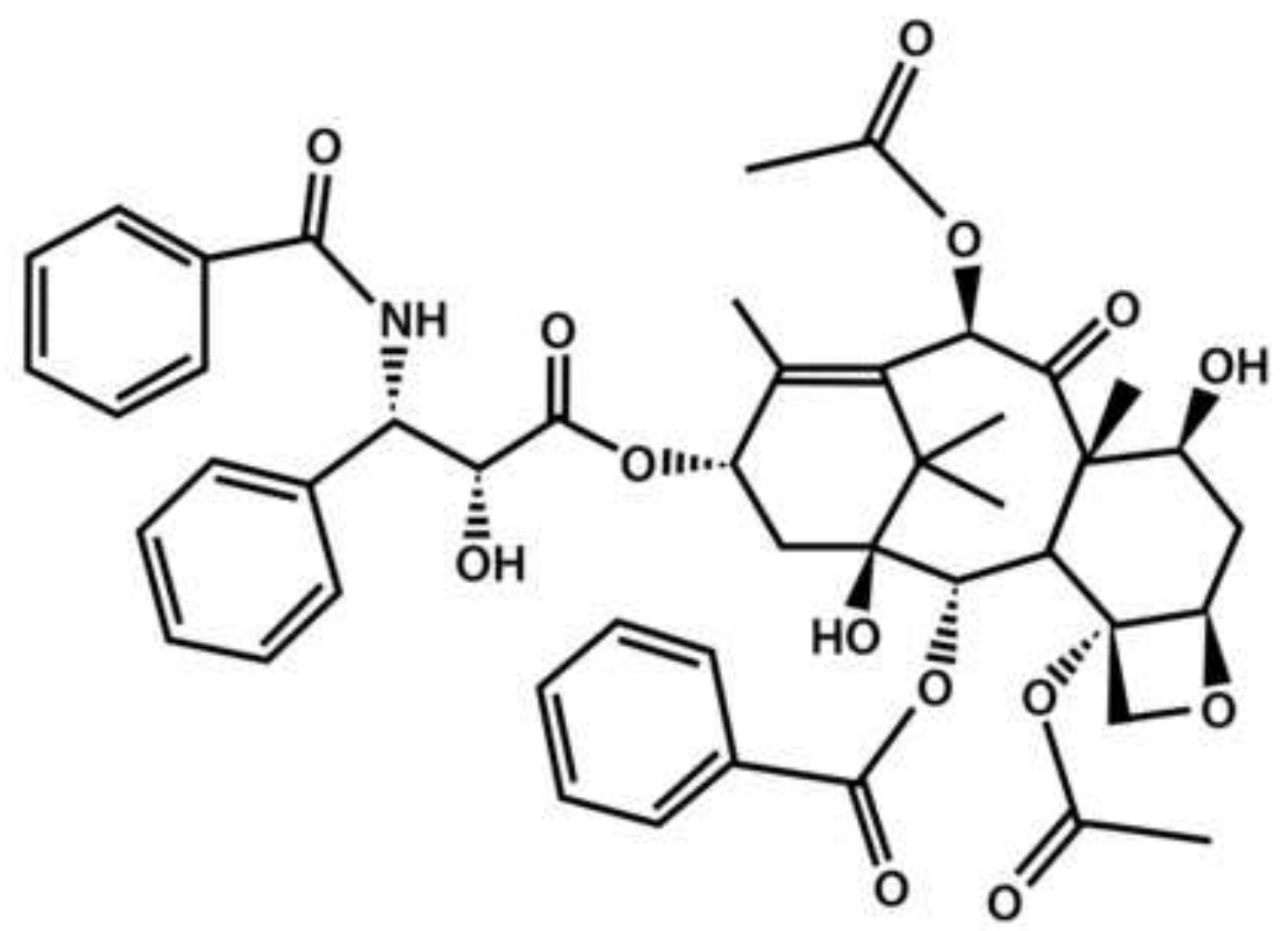


Figure 3. The Effect of Paclitaxel on hASC Viability

A.

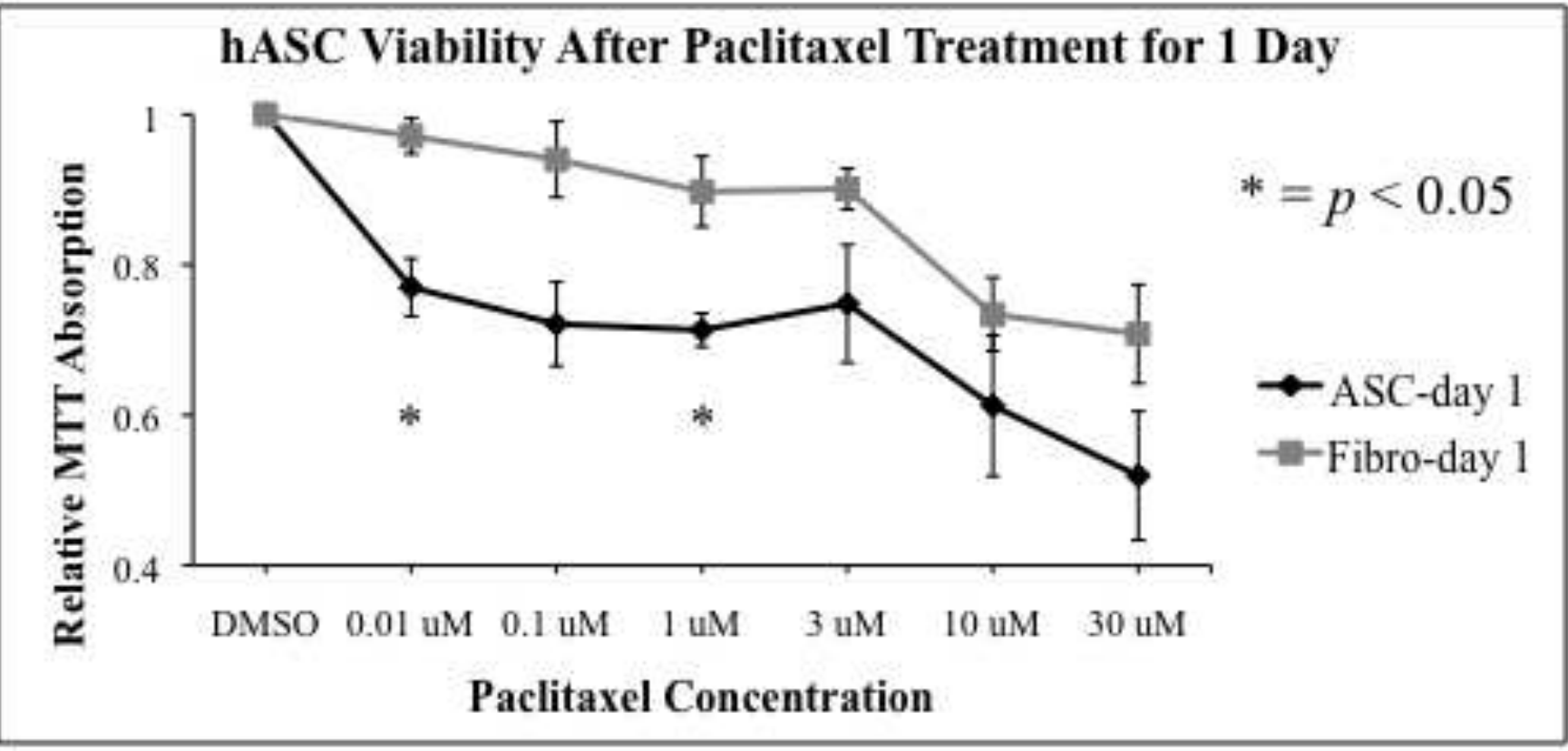

B.

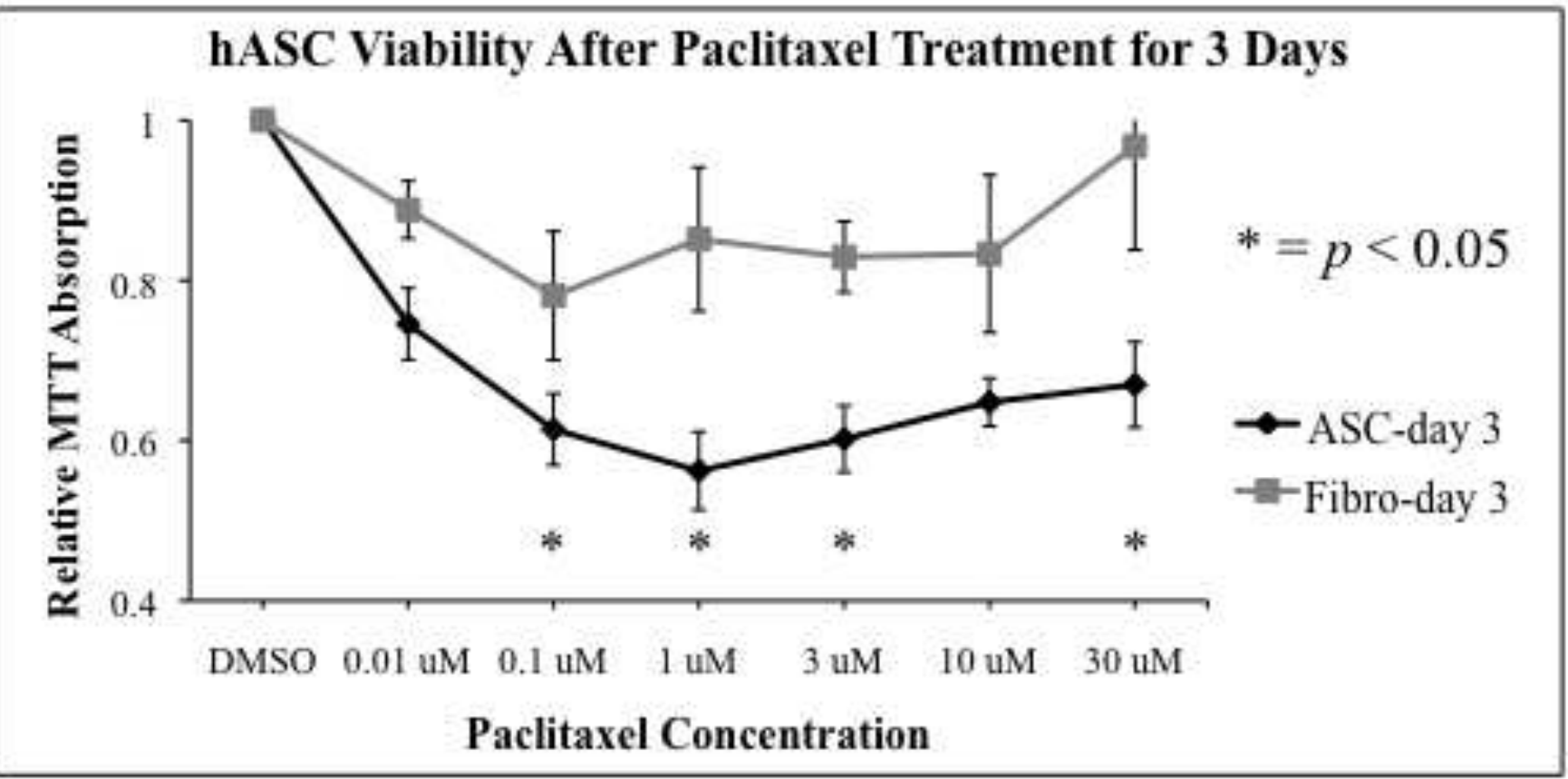


Figure 4. The Effect of Paclitaxel (PTX) on hASC Growth

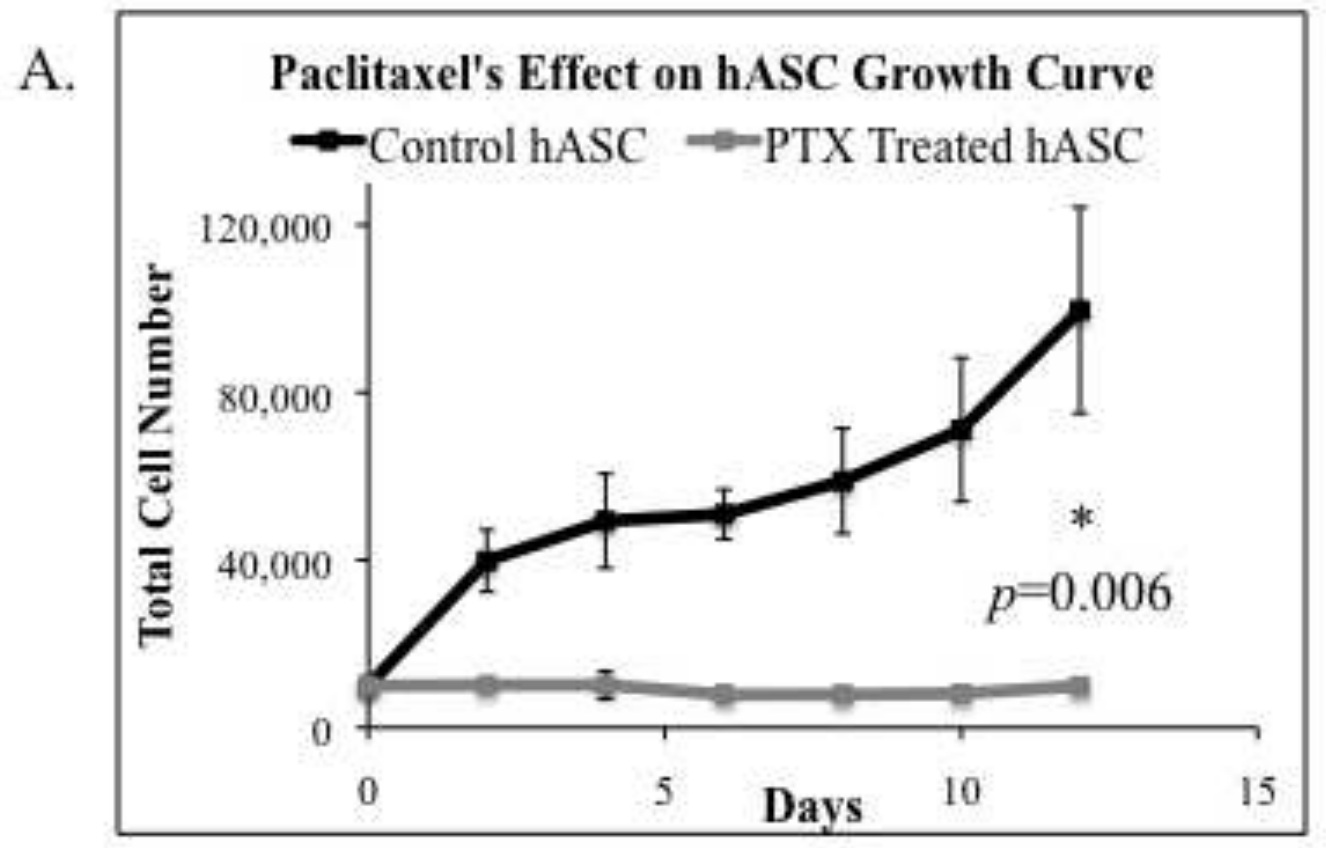

C. Paclitaxel's Effect on DNA Synthesis

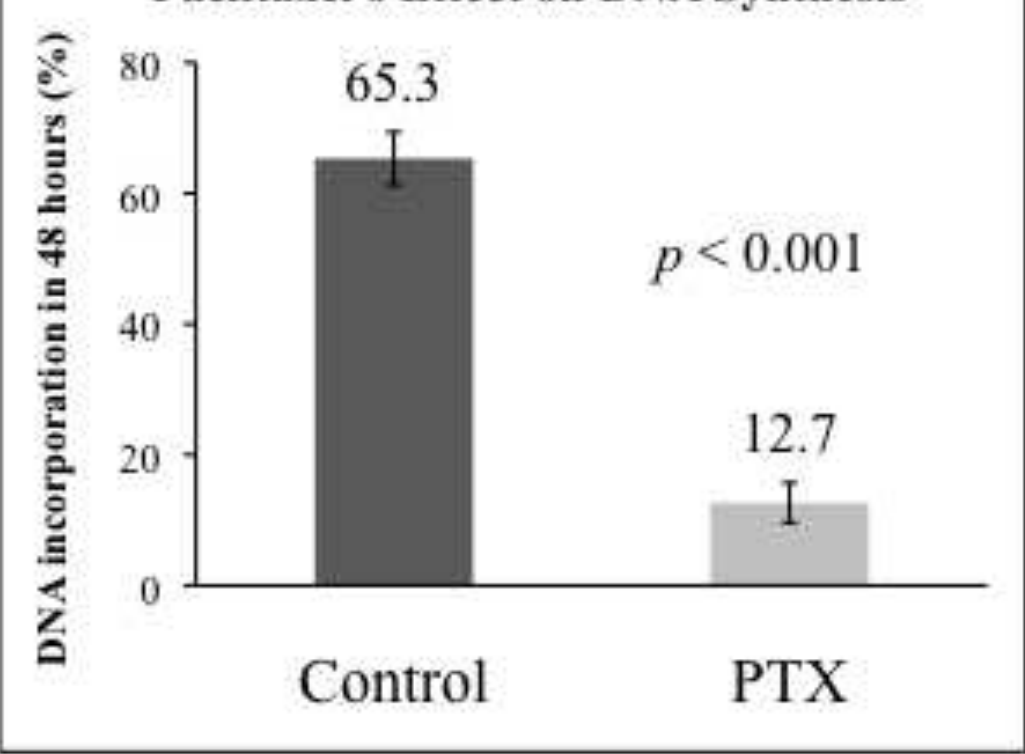

B.

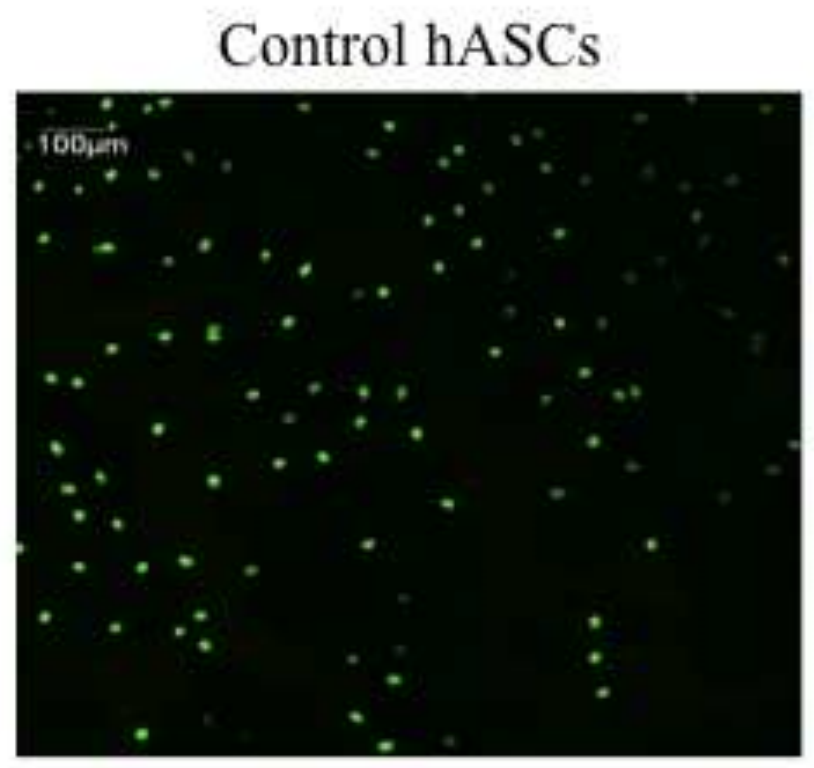

Paclitaxel Treated hASCs

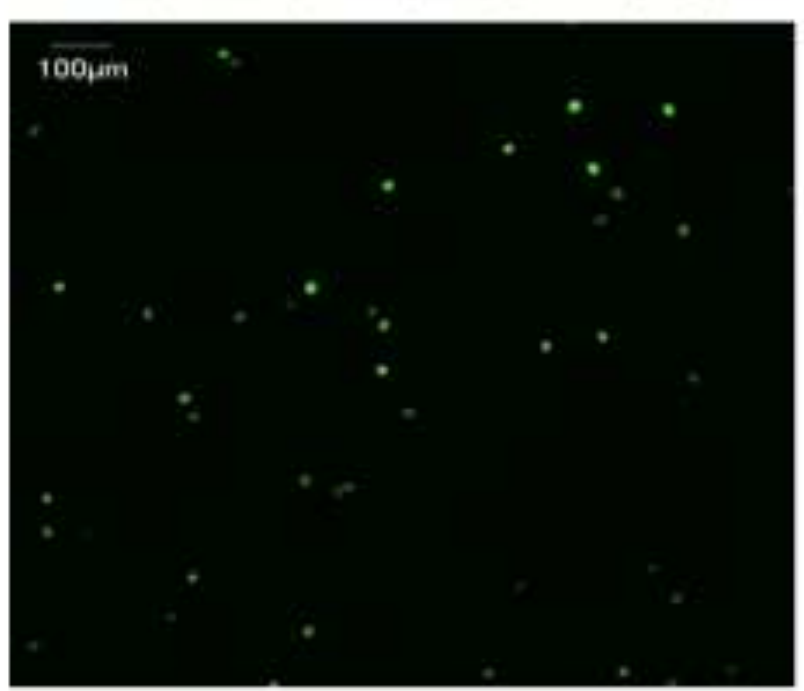




\section{Figure 5. The Effect of Paclitaxel (PTX) on hASCs} Adipogenic and Osteogenic Differentiation

A.

Oil Red O Staining PTX-

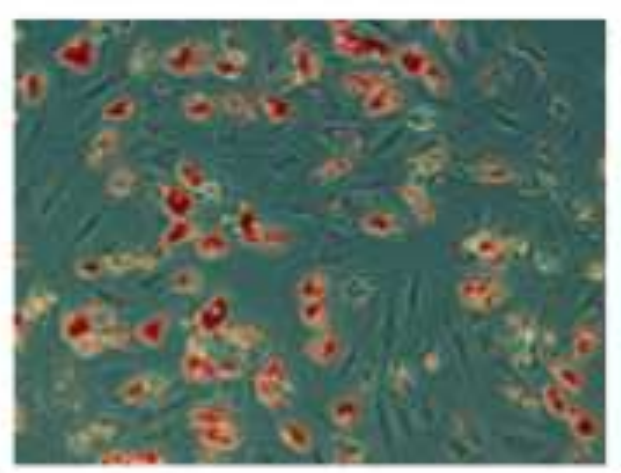

Oil Red O Staining PTX+

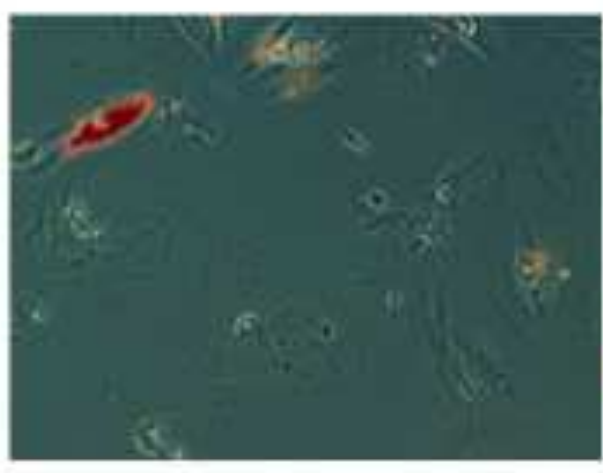

C.

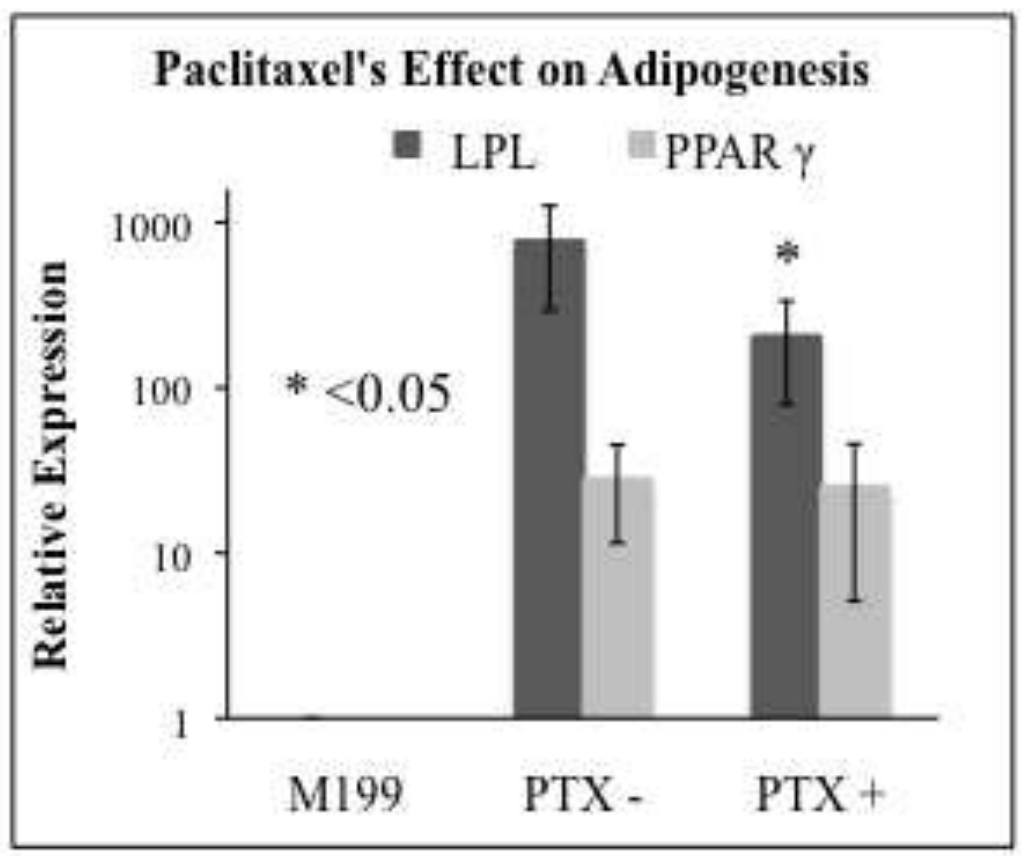

B.

Alizarin Red Staining PTX-

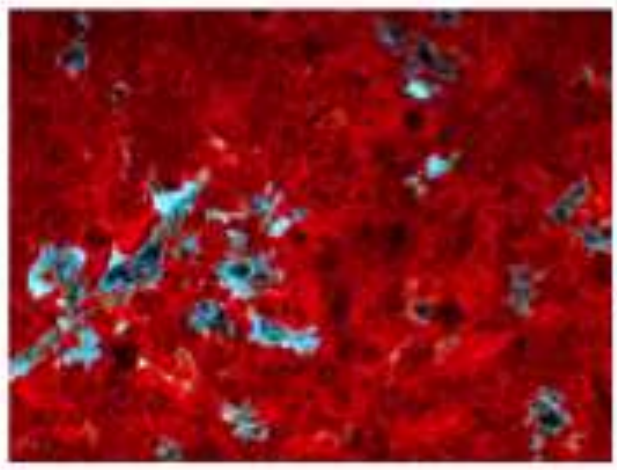

Alizarin Red Staining PTX+

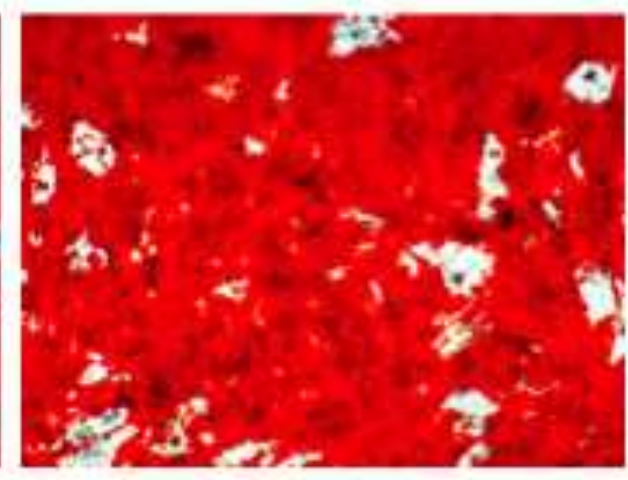

D.

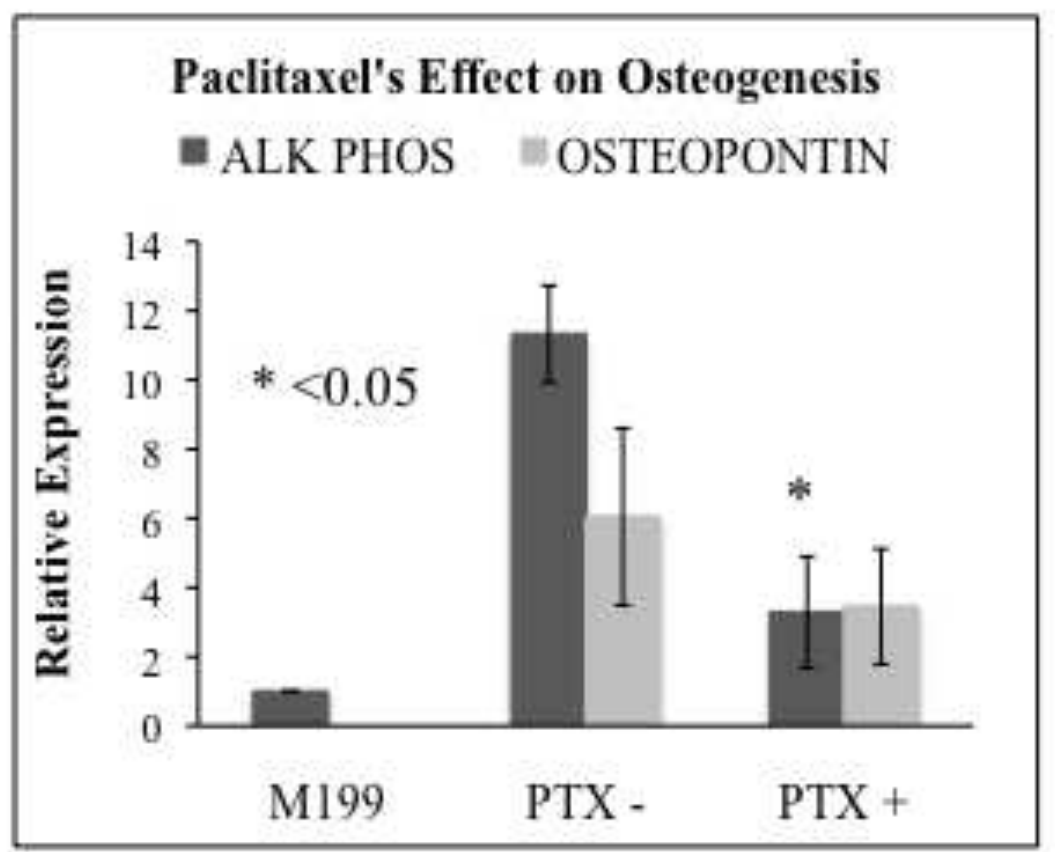


Figure 6. The Effect of Paclitaxel (PTX) on hASC Endothelial Differentiation

A.

Bright Field Cell Image Matrigel (4X) Matrigel (10X)
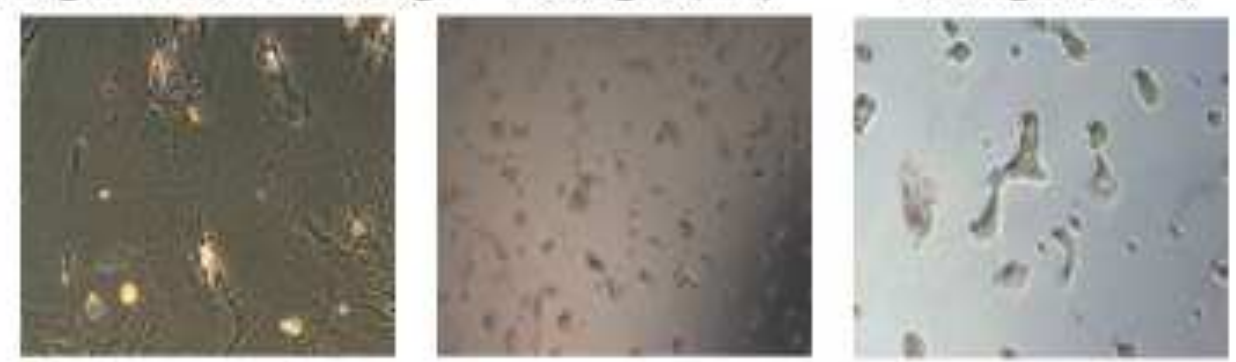

PTX +
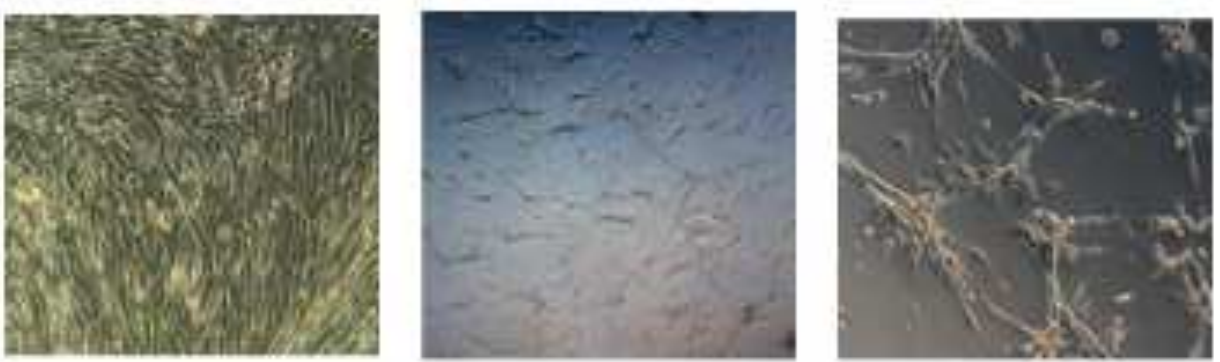

B.

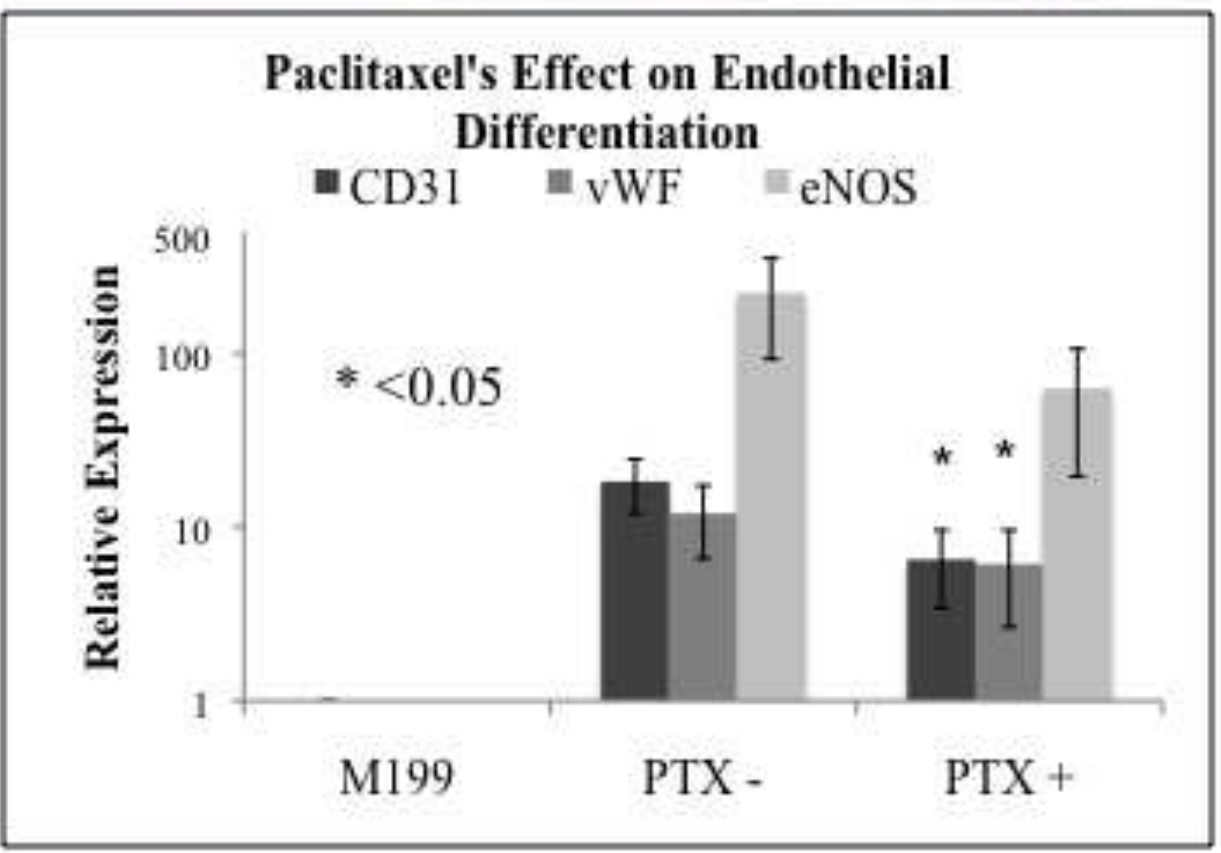


Figure 7. Annexin V Apoptosis Using Flow Cytometry to Determine Paclitaxel's (PTX) Effect on hASC Apoptosis.
A. Control (No PTX)
B. I Day PTX
C. 3 Day PTX
D. 7 Day PTX
(1 Dose PTX)
E. 7 Day PTX
Treatment
Treatment
(Continual Dose)
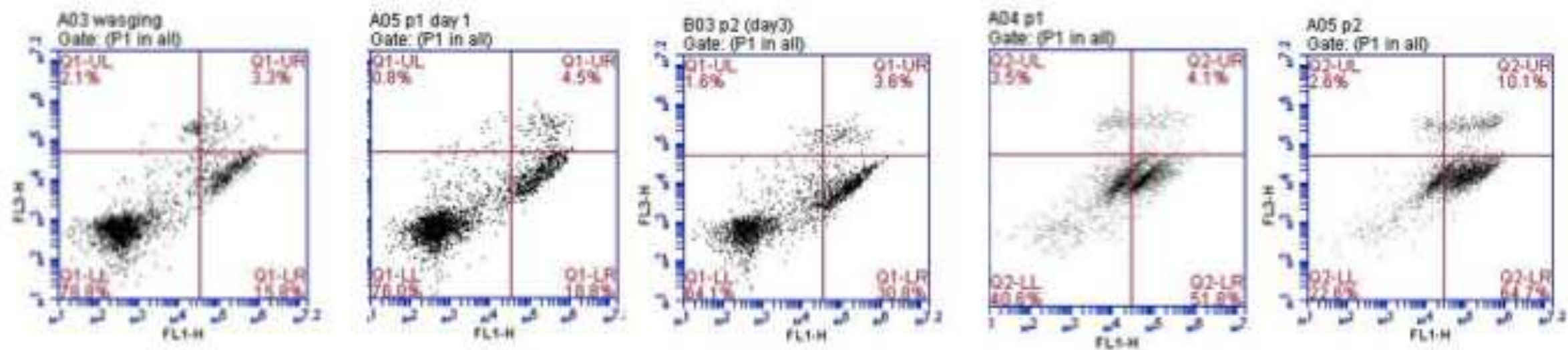
Figure 8. The Localization and Expression of TNF- $\alpha$ in Control and Paclitaxel (PTX) Treated hASCs.

A. TNF Staining for Control hASCs

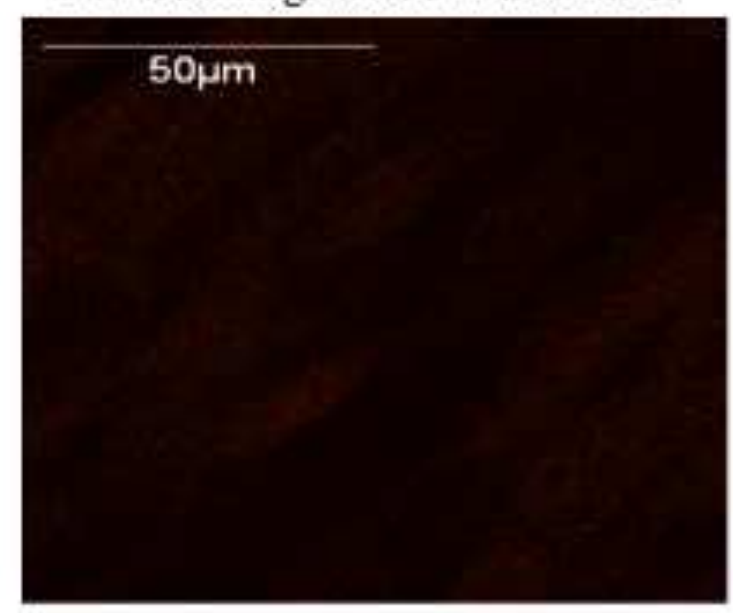

TNF Staining for PTX Treated hASCs

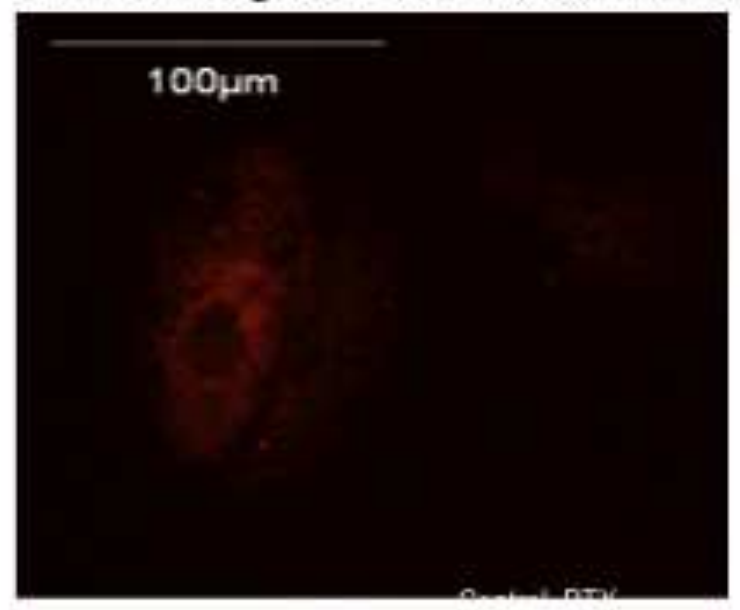

B.
$\mathrm{Cl}$
C2
PTX1 PTX2

C.

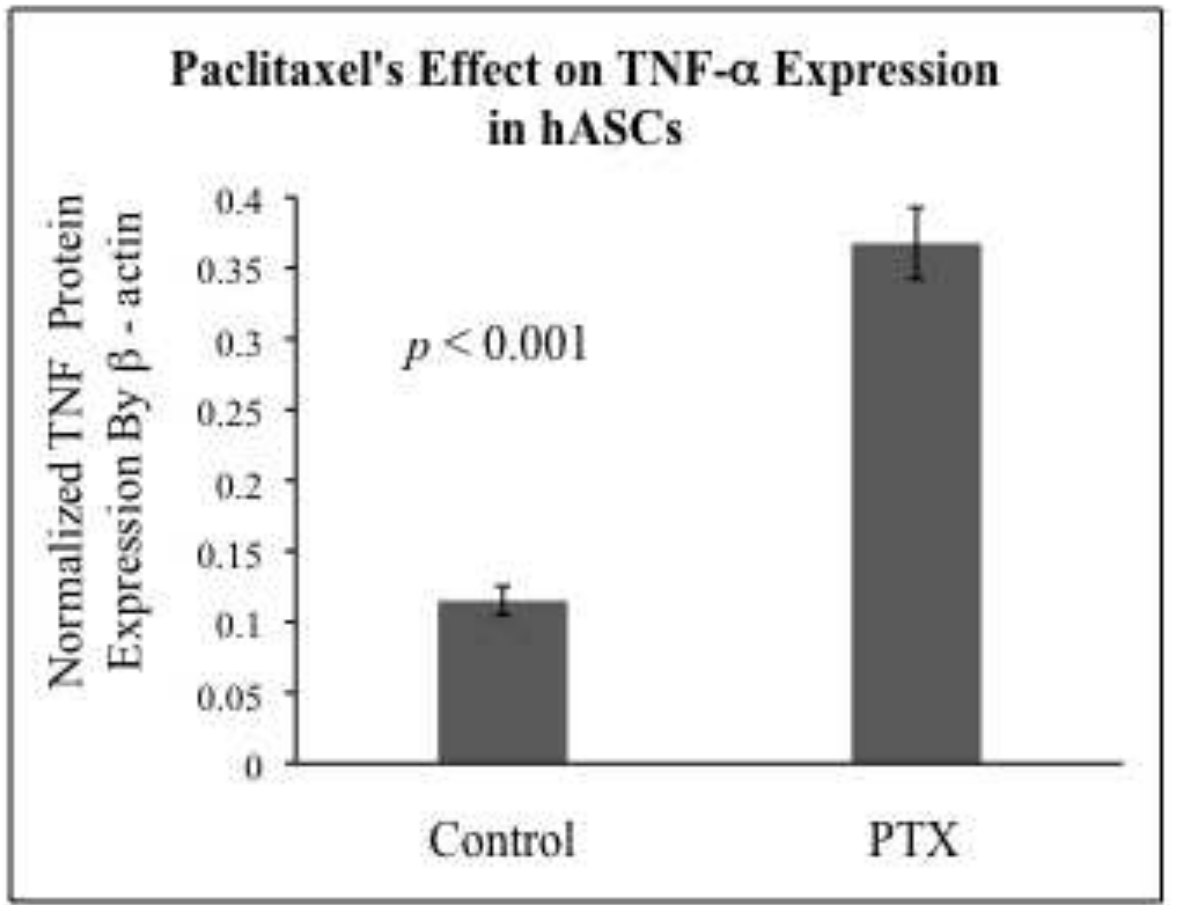

TNF

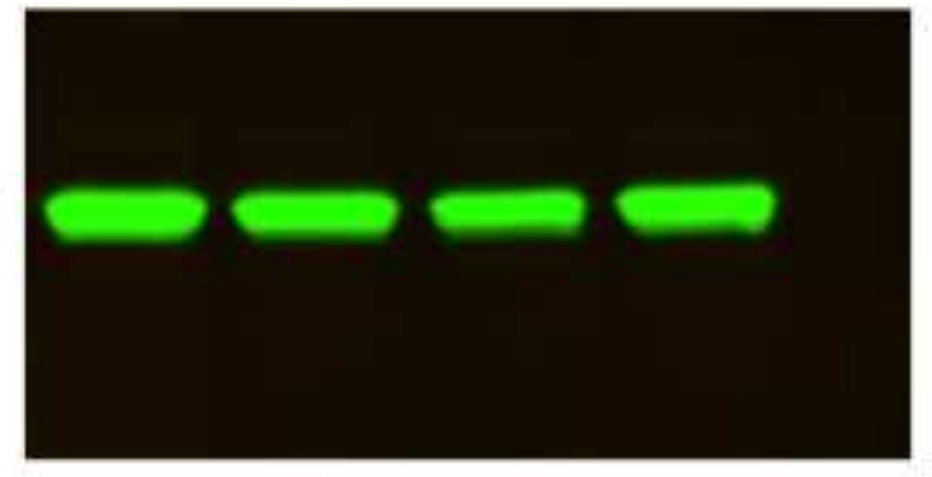

$\beta$ - actin

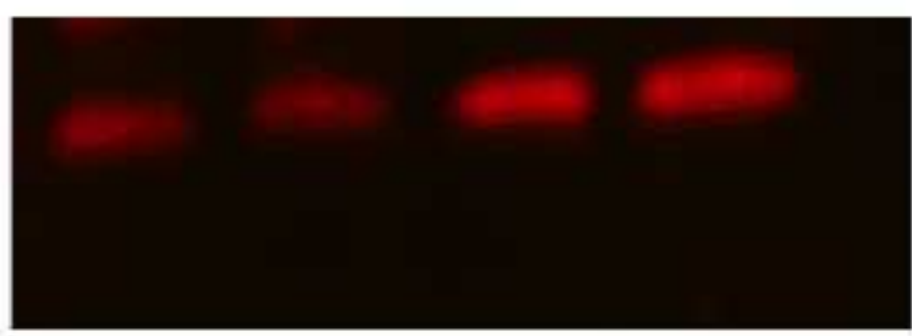

Control

\section{PTX}


Figures 9A \& B. Human Adipose Derived Stem Cell (hASC) Function With and Without Paclitaxel

A.

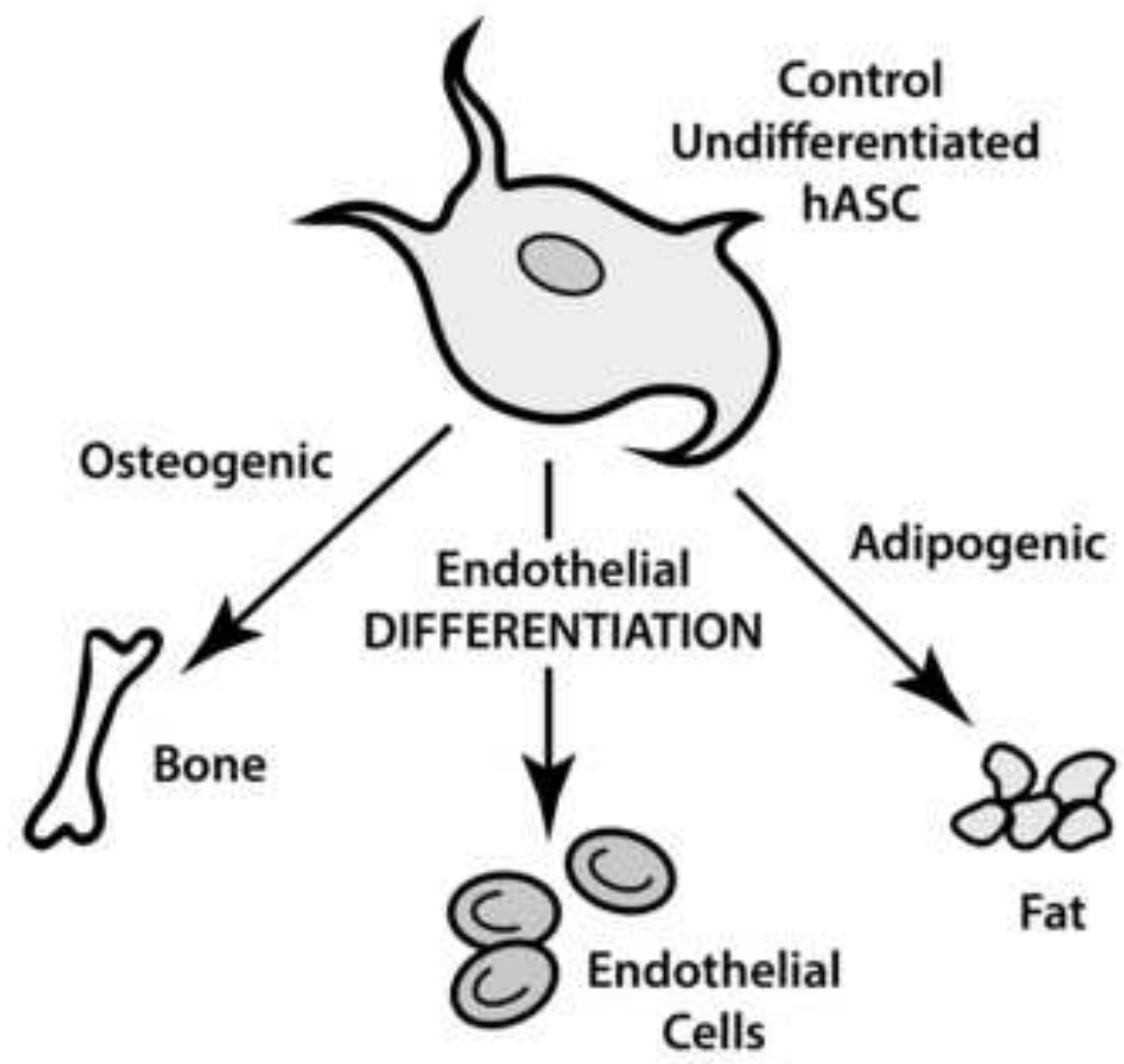

B.

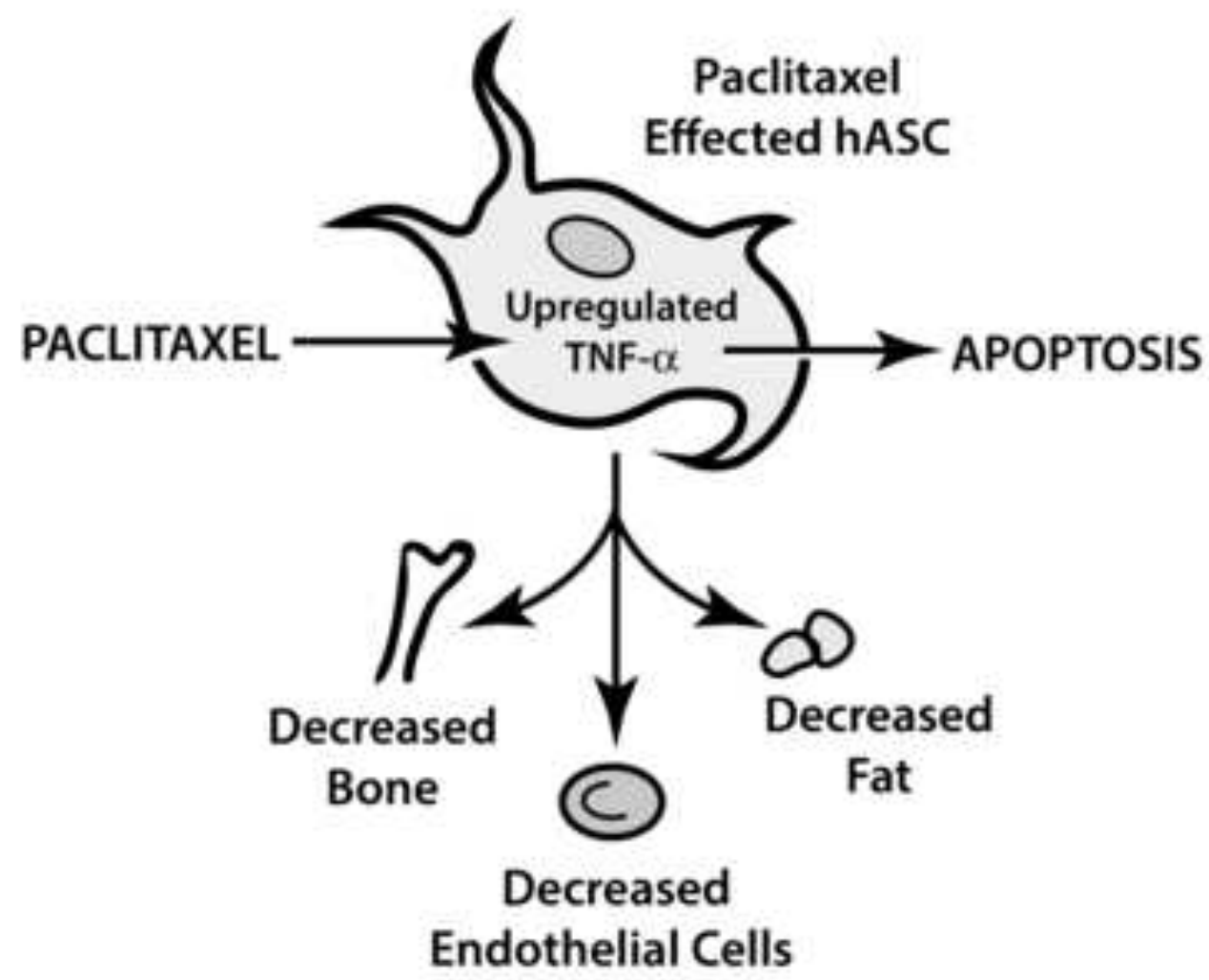

